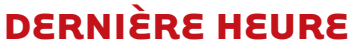

\section{Un saut symbolique mais encourageant dans le traitement de la myopathie de Duchenne}

Jean-Claude Kaplan, Jamel Chelly, Luis Garcia
J.C. Kaplan, J. Chelly:
Université Paris Descartes,

Faculté de Médecine, Institut Cochin,

24, rue du Faubourg Saint Jacques,

Paris, F-75014, France;

AP-HP, groupe hospitalier Cochin,

laboratoire de Biochimie Génétique

et Moléculaire, 123, boulevard du Port-Royal,

F75014, France.

kaplan@cochin.inserm.fr

chelly@cochin.inserm.fr

L. Garcia : UPMC-Inserm UMR S 787,

Institut de Myologie,

Faculté de Médecine Pierre et Marie Curie,

105, boulevard de l'Hôpital,

75634, Paris Cedex 13, France.

luis.garcia@chups.jussieu.fr

entité encore fonctionnelle malgré l'amputation de 20 des 24 répétitions de spectrine dans le domaine central, correspondant à une délétion en phase des exons 17 à 57 [8]. Au total, l'organisation du gène $D M D$ avec ses 79 exons dont le découpage ne coïncide pas avec celui des 3685 codons (voir Figure 1), la structure modulaire de la dystrophine avec des domaines répétés non indispensables, et le spectre mutationnel de la pathologie du gène DMD (75\% de délétions ou duplications portant sur un nombre entier d'exons; $15 \%$ de mutations ponctuelles non-sens) font de la myopathie de Duchenne un véritable cas d'école pour le SET.

Le principe directeur du SET étant de restaurer une certaine production de dystrophine tronquée chez des malades où le déficit est complet, on s'attend non pas à obtenir une guérison totale, mais au moins à convertir une DMD en BMD. C'est l'équipe de G.J. van Ommen à Leiden (Pays-Bas) qui a proposé la première ce concept d'épissothérapie, et qui en a poussé les feux avec une remarquable constance et efficacité [9-11] ce qui a abouti à la réalisation du premier essai clinique par SET [1] que nous commentons ici.

\section{Méthodologie et efficacité du SET}

- Pour réaliser un saut d'exon il faut bloquer l'intégration d'un ou plusieurs exons dans le 
transcrit final en masquant spécifiquement des séquences essentielles au déroulement normal de l'épissage. On y parvient grâce à l'apport d'oligonucléotides antisens ou AON (en général des 20-mer) électivement ciblés sur un site canonique accepteur ou donneur d'épissage, ou une séquence de type $\varepsilon S \varepsilon$, ou un site de branchement. Deux stratégies d'utilisation des AON ont déjà fait leur preuve. Les AON libres, chimiquement modifiés pour les rendre insensibles aux nucléases endogènes (par exemple les 2'-0-méthyl-phosphorothioates, ou les morpholino-phosphorodiamidates), ont l'énorme avantage de ne pas être immunogènes, mais il faut les réapprovisionner de façon itérative. Les AON non modifiés mais camouflés dans un petit ARN naturel par exemple snRNAU7. Ce véritable cheval de Troie est vectorisé dans un AAV (adenoassociated virus), ce qui assure une production permanente de l'antisens [12], mais pose le problème de l'immunogénicité du vecteur viral. L'efficacité de la stratégie du SET a été validée, tant ex-vivo (myoblastes en culture) qu' in vivo (par voie intramusculaire ou intravasculaire) sur des modèles animaux de dystrophinopathies dues à des mutations nulles (stop de la souris $m d x$; mutation d'épissage du chien GRMD) [9-15]. Les résultats ont été spectaculaires puisqu'une réexpression stable de la «quasi-dystrophine» attendue a été obtenue avec un rendement très significatif. Dans les expériences in vivo elle s'est accompagnée d'une amélioration de la force musculaire [12-15].

\section{Le passage à l'homme}

Ces indispensables preuves de principe étant obtenues on pouvait passer à l'homme. Ce sont les résultats du premiers essai clinique de phase $1^{2}$ qui sont maintenant rapportés par les membres de l'équipe de Leiden [1]. Le choix de l'exon s'est porté

${ }^{2}$ On y vérifie l'innocuité, à la rigueur l'efficacité biologique, mais pas encore l'effet thérapeutique.

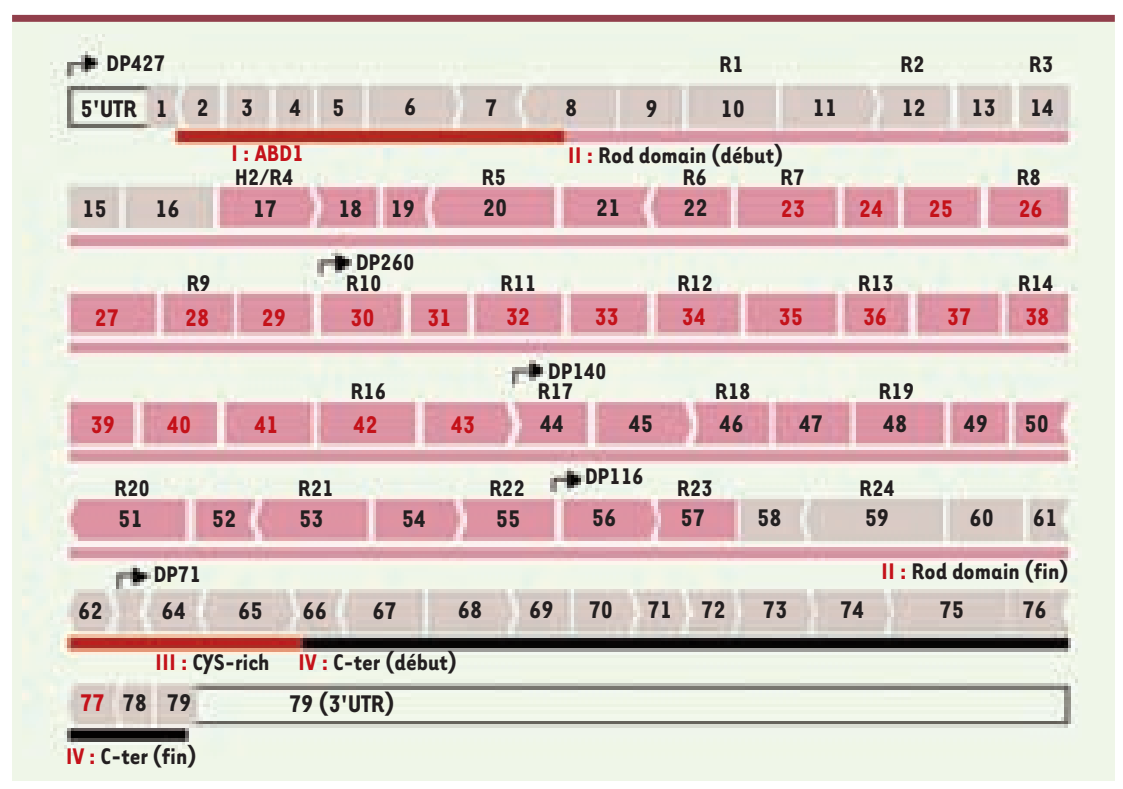

Figure 1. Phasage des exons du gène DMD et correspondance avec les domaines de la dystrophine. L'articulation des exons ne coïncide pas nécessairement avec l'enchaînement des codons dont certains peuvent être interrompus par un intron:

mode $3-1$ : entre 2 codons
mode $1-2$ : entre le $1^{\text {er }}$ et le $2^{\mathrm{e}}$ nucléotide d'un même codon $2-3$ : après le $2^{\mathrm{e}}$ et le $3^{\mathrm{e}}$ nucléotide d'un même codon

(extrait de [2], avec l'autorisation de Flammarion Médecine-Sciences).

sur l'exon 51, car sa non-inclusion dans le transcrit final est capable de restaurer le cadre de lecture dans au moins 6 patrons de délétions hors-phase: del-50, del-52, del 49-50, del-48-50, del 47-50, del 4550 représentant cumulativement $25 \%$ des DMD délétionnelles. De plus, les transcrits obtenus par SET de l'exon 51 devraient avoir une excellente fonctionnalité, car dans les bases de données de dystrophinopathies les sujets trouvés porteurs du patron corrigé par exclusion du 51 sont très rares. Dans notre propre expérience à l'hôpital Cochin sur 232 cas de BMD non apparentés seuls 7 cas ont une délétion correspondant à un patron de délétion similaire à celui qui résulterait d'un SET de l'exon 51 (4 del 48-51, 2 del 45-51, 1 del 49-51). Les 3 autres patrons n'ont pas été trouvés, et on peut considérer soit qu'ils ne se produisent pas spontanément, soit qu'ils sont asymptomatiques, cette dernière hypothèse paraissant la plus vraisemblable ${ }^{3}$.

Dans l'essai thérapeutique rapporté par van Deutekom et al. [1] 4 myopathes de Duchenne de 10 à 13 ans, ayant déjà perdu la marche et portant chacun une délétion différente corrigible par SET de l'exon 51 (del 50, del 48-50, del 49-50 et del 52) ont été sélectionnés. Après vérification de l'efficacité de la procédure sur leur myoblastes (apparition du transcrit attendu et de la dystrophine), les malades ont reçu une injection intramusculaire dans le muscle tibial antérieur de 0,8 mg de PR005l (laboratoire Prosensa), un AON 20-mer de type 2'-0-méthyl phosphorothioate conçu pour masquer par hybridation un ESE situé eu milieu de l'exon 51. Le territoire musculaire ayant reçu l'injection a été biopsié le $28^{\mathrm{e}}$ jour, pour une analyse dans des coupes sériées des transcrits et de la dystophine (par immunofluorescence et par Westernblot). Dans tous les cas une proportion importante des transcrits ne portait plus l'exon 51 , et la dystrophine était présente en quantités comprises entre 3 et $12 \%$ de la normale. Le territoire corrigé était trop res-

\footnotetext{
${ }_{3}^{3}$ Fait intéressant nous avons trouvé fortuitement un cas unique de del51-52 cliniquement asymptomatique (résultat personnel non publié).
} 
treint pour pouvoir explorer une quelconque récupération de la force musculaire. Aucune réaction indésirable, locale ou générale, n’a été observée.

\section{Quelle est la portée de ces résultats?}

Elle est d'une portée médicale considérable. Certes la preuve de principe expérimentale du SET de l'exon 51 avait déjà été apportée ex vivo dans des cellules souches musculaires (dites $\mathrm{C} 33^{+}$) d'un myopathe de Duchenne porteur d'un délétion 49-50 [16]. Mais il s'agit maintenant d'une expérimentation effectuée in vivo chez l'homme, un passage difficile à franchir psychologiquement, médicalement et règlementairement. On ne peut que saluer le travail de l'équipe de Leiden, qui est l'aboutissement de près de 10 années d'effort, ayant comporté successivement la conception et l'optimisation de la méthode de SET par AON chimiquement modifiés, la validation rigoureuse de l'efficacité et de l'innocuité chez l'animal, la mise en œuvre d'une plate-forme pour la synthèse et le conditionnement de grandes quantités d'AON pharmacologiquement conformes par une Biotech (Prosensa). Cet investissement scientifique et logistique commence aujourd'hui à porter ses fruits. Mais, si ce premier essai clinique est très encourageant, on ne saurait se contenter d'une administration très localisée du produit thérapeutique. La prochaine étape consiste à opérer en grandeur nature, c'està-dire à passer à une voie systémique, seule susceptible d'atteindre un nombre suffisant de muscles pour obtenir l'efficacité clinique. Le véritable enjeu est d'enrayer le processus dystrophique dans des muscles essentiels pour la qualité de la vie et pour les fonctions vitales (diaphragme, cœur). Pour cela on n'est pas encore prêt car de nombreuses questions restent pendantes. Quelles sont les meilleures formes galéniques? Quelle est la posologie et la périodicité optimales? Quelle est la tolérance à long terme des AON dont on sait qu'ils ne sont pas métabolisés? Quels sont les risques d'atteindre d'autres cibles non visées? Enfin se pose la question de l'âge auquel il faut entreprendre le traitement? A priori, il faudrait intervenir aussi précocement que possible avant que les muscles ne deviennent par trop dystrophiques. Dans cet ordre d'idée, il est significatif que dans ce premier essai clinique le meilleur résultat, exprimé en taux de dystrophine, ait été obtenu chez le patient dont le muscle était le moins dystrophique. Pour atteindre le plus grand nombre de malades d'autres exons doivent pouvoir être ciblés. Théoriquement trois quarts des DMD devraient pouvoir bénéficier du SET. On sait maintenant restaurer la phase par des sauts multiples d'exons ce qui augmente considérablement le nombre de patrons délétionnels corrigibles $[10,11]$. On a calculé que deux tiers d'entre eux pourraient en théorie bénéficier d'un SET «passe-partout » consistant à obtenir l'exclusion des exons 45 à 55 [17].

En fait il faut mener de front les différentes pistes qui s'offrent désormais et que nous avons évoquées plus haut. Déjà un autre essai clinique utilisant une autre chimie des AON (les morpholino) est en cours à Londres. Le SET, de même que le forçage des nonsens directs par des agents de translecture comme le PTC $124^{4}$ [18] s'inscrivent dans le cadre de ce qu'il faut appeler une thérapie spécifique d'allèle $e^{5}$. Ceci impose de caractériser la mutation chez chaque malade, non seulement dans un but diagnostique, mais aussi dans une perspective thérapeutique. Les progrès futurs en matière de recherche et d'essais cliniques dépendent de la prise de conscience de ce qui apparaît désormais comme un devoir moral. $\diamond$ Effective exon skipping in humans: a symbolic but promising jump into DMD therapy

\section{RéFÉRENCES}

1. Van Deutekom JC, Janson AA, Ginjaar IB, et al. Local dystrophin restoration with antisense oligonucleotide PR0051. N Engl J Med 2007 ; $357: 2677-86$.

2. Kaplan JC, Delpech M. Biologie moléculaire et médecine, $3^{\mathrm{e}}$ ed. Paris : Flammarion MédecineSciences, $2007:$ 401-16.

\footnotetext{
${ }^{4}$ Avec lequel des essais cliniques sont également commencés.

${ }^{5}$ À ne pas confondre avec la médecine dite personnalisée qui prétend soigner les individus sur la base de leur caractéristiques génomiques globales.
}

3. Van Deutekom JC, Van Ommen G. Advances in Duchenne muscular dystrophy gene therapy. Nat Med $2003 ; 4: 774-83$.

4. Goyenvalle A, Vulin A, Fougerousse F, et al. Le saut d'exon thérapeutique: un espoir pour les dystrophinopathies. Med Sci (Paris) 2004 ; 20: 1163-5.

5. Benchaouir R, Meregalli M, D’Antonio G, et al. Réhabilitation de la dystrophine humaine dans le modèle scid/mdx après greffe de cellules souches de patients DMD corrigées par saut d'exon. Med Sci (Paris) 2008; $24: 99-101$.

6. Monaco AP, Bertelson CJ, Liechti-Gallati S, et al. An explanation for the phenotypic differences between patients bearing partial deletions of the DMD locus. Genomics $1988 ; 2: 90-5$.

7. England S, Nicholson L, Johnson M, et al. Very mild muscular dystrophy associated with the deletion of $46 \%$ of dystrophin. Nature 1990 ; 343 : 180-2.

8. Harper SQ, Hauser MA, DelloRusso C, et al. Modular flexibility of dystrophin: implications for gene therapy of Duchenne muscular dystrophy. Nat Med $2002 ; 8: 253-61$.

9. Van Deutekom JC, Bremmer-Bout M, Janson AA, et al. Antisense-induced exon skipping restores dystrophin expression in DMD patient derived muscle cells. Hum Mol Genet 2001 ; 10 : 1547-54.

10. Aartsma-Rus A, Janson AA, Kaman WE, et al. Antisense-induced multiexon skipping for Duchenne muscular dystrophy makes more sense. Am J Hum Genet $2004 ; 74: 83-92$

11. Aartsma-Rus A, Kaman WE, Weij R, et al. Exploring the frontiers of therapeuticexon skipping for Duchenne muscular dystrophy by double targetingwithin one or multiple exons. Mol Ther 2006 ; $14: 401-7$.

12. Goyenvalle A, Vulin A, Fougerousse F, et al. Rescue of dystrophic muscle through U7 snRNA-mediated exon skipping. Science 2004 ; 306 : 1796-9.

13. Lu QL, Rabinowitz A, Chen YC, et al. Systemic delivery of antisense oligoribonucleotide restores dystrophin expression in body-wide skeletal muscles. Proc Natl Acad Sci USA 2005 ; 102 : 198-203.

14. McClorey G, Moulton HM, Iversen PL, et al. Antisense oligonucleotide-induced exon skipping restores dystrophin expression in vitro in a canine model of DMD. Gene Ther 2006 ; 13 : 1373-81.

15. Alter J, Lou F, Rabinowitz A, et al. Systemic delivery of morpholino oligonucleotide restores dystrophin expression bodywide and improves dystrophic pathology. Nat Med 2006 ; 12 : 175-7.

16. Benchaouir R, Meregalli M, Farini A, et al. Restoration of human dystrophin following transplantation of exon-skipping-engineered DMD patient stemcells into dystrophic mice. Cell Stem Cell $2007 ; 1: 644-57$.

17. Béroud C, Tuffery-Giraud S, Matsuo M, et al. Multiexon skipping leading to an artificial DMD protein lacking amino acids from exons 45 through 55 could rescue up to $63 \%$ of patients with Duchenne muscular dystrophy. Hum Mutat 2007 ; $28: 196-202$.

18. Welch EM, Barton ER, Zhuo J, et al. PTC124 targets genetic disorders caused by nonsense mutations. Nature 2007 ; 447 : 87-91.

\section{TIRÉS À PART}

J.C. Kaplan 
Médecine/Sciences est une revue internationale mensuelle francophone d'information dans tous les domaines de la biologie et de la médecine. Elle traite des principales découvertes, des sujets d'actualité, qu'il s'agisse de nouvelles technologies, de progrès thérapeutiques, ou de l'apparition de pathologies nouvelles dans le monde.

$\varepsilon n$ écrivant dans $m / s$, les auteurs voient leurs articles référencés dans PubMed, mais ont, de surcroît, le plaisir de faire partager aux lecteurs, dans leur langue, leur intérêt et parfois leur enthousiasme, pour tel ou tel sujet, en y apportant leur touche d'humour et de culture, surtout dans la partie Forum, où la plus grande liberté d'expression est autorisée.

Toutefois, comme la revue s'adresse à un lectorat très varié de scientifiques confirmés, d'enseignants, d'étudiants et de médecins, elle implique de la part des auteurs de développer leurs sujets, en allant jusqu'au bout des connaissances scientifiques, quelle qu'en soit la difficulté. Cette recommandation est particulièrement dédiée aux Synthèses, qui ont pour ambition de faire le point sur un sujet donné, mais aussi d'adopter un style aussi clair et accessible que possible, quel que soit le niveau technique ou théorique de leur propos, afin d'être intelligible par les non spécialistes.

La rédaction de la revue demande à tout auteur, sollicité ou proposant spontanément un manuscrit, de se conformer à quelques règles qui ne pourront qu'en faciliter l'évaluation et, une fois celui-ci accepté, d'en hâter la publication :

- écarter autant que faire se peut tous les mots anglais et éviter les anglicismes, tant sur le plan du vocabulaire que de la syntaxe;

- toujours définir les acronymes ;

- rassembler en tableaux, glossaires, les précisions techniques, méthodologiques, les termes peu répandus et les compléments d'information qui surchargeraient le texte;

- respecter la longueur du manuscrit et le nombre de références;

- deux à trois références d'articles déjà publiés dans $\mathrm{m} / \mathrm{s}$ seront très appréciées.

\section{Les rubriques de $\mathrm{m} / \mathrm{s}$}

Pour le format et la présentation des articles, médecine/sciences propose trois formules.

1. La partie Revues présente des Synthèses qui font le point sur un sujet par un auteur spécialiste du domaine. Les synthèses véhiculent une pensée, un esprit critique, un message, au-delà du catalogue des faits collectés sur un sujet. Elles doivent permettre une vraie discussion des résultats scientifiques.

Cette partie comporte également des Dossiers techniques (exposé d'une technique ou d'un ensemble de techniques susceptible(s) de favoriser le développement de recherches en sciences biomédicales).

2. La partie Forum propose des Faits et Chiffres dans le domaine de l'épidémiologie, de la démographie, de l'économie de la santé..., et des articles de réflexion, c'est-à-dire, des Perspectives et des Chroniques sur des sujets faisant l'objet de débats dans la communauté scientifique, ainsi que des revues sur l'Histoire biomédicale, les Sciences sociales et la santé, la Santé et l'environnement, entre autres.

3. La partie Magazine est le reflet de l'actualité scientifique, faisant état, dans des textes courts, de résul- tats originaux importants récemment publiés. Elle est constituée soit de Nouvelles, spontanées ou sollicitées, soit de Brèves, courtes notes de lecture. Tous les articles de $\mathrm{m} / \mathrm{s}$ sont signés par leurs auteurs

\section{Normes générales de présentation des articles}

Textes et tableaux adressés en fichiers Word, PC ou Macintosh (enregistrements en .doc, format PDF exclus) - illustrations en fichiers séparés. Tableaux et illustrations appelés dans le texte.

Illustrations: schémas en format Illustrator ou PowerPoint, photos en format jpeg ou tif. Lorsque nécessaire, l'échelle de l'image doit apparaître dans l'illustration et sa valeur être indiquée dans la légende. Légendes complètes et détaillées des figures et tableaux.

Illustrations numérotées en chiffres arabes (ex : figure 1) et tableaux en chiffres romains (ex: tableau II).

Important : les auteurs sont priés de mentionner tout conflit d'intérêt potentiel concernant l'article soumis à publication dans $\mathrm{m} / \mathrm{s}$, en particulier de nature financière. Cette information sera gardée confidentielle par la rédaction de $\mathrm{m} / \mathrm{s}$ jusqu'à la publication de l'article.

\section{Les Synthèses}

Elles ne peuvent excéder 18000 caractères (espaces compris, références exclues), 30 références et 3 à 4 illustrations (figures et tableaux), avec un titre en français et en anglais.

Elles doivent être accompagnées d'un texte d'environ 700 caractères destiné à offrir un aperçu rapide du sujet et à susciter l'intérêt du lecteur (chapô) ; celui-ci figurera en caractères gras en tête de l'article. Un résumé en anglais d'environ 1000 caractères doit être fourni, nécessaire à l'indexation de l'article dans PubMed.

Un encadré «Prise de distance » de 1000 à 1500 caractères pourra souligner les implications conceptuelles ou méthodologiques posées par les résultats et les stratégies futures pour les résoudre.

\section{Présentation des références}

Appelées dans le texte par leurs numéros entre crochets ([1], [2], [3-5]) et classées par ordre d'apparition dans l'article.

Pour éviter toute redondance, alléger les textes et souligner la dynamique des connaissances, il est recommandé aux auteurs de rechercher et de mentionner les articles parus précédemment dans médecine/sciences sur le sujet.

Tous les noms des auteurs sont mentionnés, suivis des initiales de leurs prénoms, jusqu'au nombre de quatre. Au delà, les trois premiers le sont, suivis de et al. (en italique).

\section{Pour les articles de revues}

Exemple : Sivori S, Falco M, Della Chiesa M, et al. CpG and double-stranded RNA trigger human NK cells by Toll-like receptors: induction of cytokine release and cytotoxicity against tumors and dendritic cells. Proc Natl Acad Sci USA 2004; 101: 10116-21.

\section{Pour les articles d'ouvrages}

Exemple: Ménard D, Beaulieu JF, Boudreau F, et al. Gastrointestinal tract. In: Unsicker K, Krieglstein K, eds. Cell signaling and growth factors. New York: Wiley, 2005: 755-90.
Pour les ouvrages

Exemple : Kupiec JJ, Sonigo P. Ni Dieu ni gène. Paris : Seuil, $2004: 230 \mathrm{p}$.

\section{Les Nouvelles}

6000 caractères au maximum (espaces compris, références exclues), 10 références au plus et 1 à 2 figures. Un titre en français et en anglais.

Présentation des références identique à celle des Synthèses (voir plus haut).

\section{Les Brèves}

2000 caractères au maximum (espaces compris).

Exemple de référence : Chneiweiss $\mathrm{H}$, et al. Med Sci (Paris) $2002 ; 18: 1065-75$

Tous les articles doivent être accompagnés des coordonnées de tous les auteurs: • nom et prénom, • institution, - adresse professionnelle, · téléphone, télécopie et courriel.

\section{Contacts :}

Rédaction Paris : contact@medecinesciences.org Rédaction Puébec : medecine.sciences@bellnet.ca

SOUMISSION ÉLECTRONIPUE MÉDECINE/SCIENCES Médecine/Sciences est dotée d'une gestion éditoriale automatisée, via le système informatique Fontisworks (http://msc.fontismedia.com). Tous les manuscrits, Éditoriaux, Synthèses, Brèves, Nouvelles, Forum, doivent être soumis par voie électronique, et nos experts devront également soumettre leur évaluation par voie électronique.

La marche à suivre est très simple : le nouvel utilisateur accède à la page d'accueil du site de soumission en ligne de Médecine/Sciences à l'adresse suivante : http://msc. fontismedia.com et clique sur le bouton «accès auteur » (ou « accès expert ») dans la liste de liens figurant sur l'écran qui s'affiche. Si l'utilisateur est un auteur, il sera d'abord invité à créer son compte en s'enregistrant. II recevra un mail de confirmation contenant son mot de passe. L'enregistrement ne s'effectue qu'une seule fois, lors de la toute première utilisation. À chaque connexion suivante, il suffit de cliquer directement sur « auteur 》 pour s'identifier, saisir le nom d'utilisateur (mail) et le mot de passe pour entrer dans le système. Une fois dans le système, l'auteur souhaitant soumettre un manuscrit suit le cheminement indiqué pour saisir les différentes informations afférant à la soumission, ainsi que pour télécharger les fichiers de son manuscrit.

Les experts, eux, seront d'abord sollicités par mail, et devront, lors d'une première étape, accepter ou refuser l'expertise en entrant dans le système via «l'accès expert», en indiquant l'identifiant (adresse e-mail) et le numéro du manuscrit $\left(\mathrm{msc}+\mathrm{N}^{\circ}\right)$ qui leur aura été indiqué dans le mail de sollicitation. Puis, comme précédemment, suivre les informations pour télécharger le manuscrit à évaluer, puis, dans un second temps, déposer leur expertise. Tous les documents nécessaires à la soumission en ligne sont accessibles sur la page de garde du site M/S de Fontismedia.

Les auteurs qui ne pourraient pas soumettre leur manuscrit via Fontismedia auront la possibilité de le soumettre par e-mail au secrétariat de Médecine/ Sciences : secretariat@medecinesciences.org

Toute information complémentaire et toute aide pourrontêtre apportées par le secrétariat de $\mathrm{M} / \mathrm{S}$ (secretariat @medecinesciences.org) (Tél : 01556413 93). 